\title{
L’égalité réelle et la mise en œuvre intégrale du principe de Jordan
}

\section{Anne Levesque*}

Cet article porte sur les problèmes et les retards dans la mise à œuvre du principe de Jordan au sein du gouvernement du Canada. Premièrement, l'article passe en survol les allégations de discrimination mises de l'avant par Assemblée des Premières Nations et la Société de soutien à l'enfance et aux familles des Premières Nations dans leur plainte de droits de la personne contre le Canada en ce qui a trait aux conflits de compétence et du manque de coordination entre les différents paliers et ministères de gouvernements et leur impact néfaste sur les enfants des Premières Nations. Deuxièmement, il résume la décision initiale du Tribunal canadien des droits de la personne [TCDP], rendue en janvier 2016, concernant le principe de Jordan. En troisième lieu, l'article examine les problèmes et les retards liés à la mise en œuvre de la décision du TCDP. Quatrièmement, il aborde en détail l'ordonnance de mai 2017 du TCDP obligeant le Canada de prendre des mesures concrètes pour se conformer à sa décision initiale. Dans la cinquième partie, l'article avance la thèse selon laquelle, qu'afin de véritablement assurer l'égalité réelle dans la société canadienne, le Canada doit être plus proactif dans l'identification et la remédiation de ses pratiques discriminatoires. En l'occurrence, la mise en auvre du "Plan l'Ourson Spirit » au sein du gouvernement canadien offre une voie prometteuse vers la fin des iniquités dans la prestation de services publics pour les enfants des Premières Nations.

This paper deals with the problems and delays that have occurred in the implementation of Jordan's Principle within the Government of Canada. The paper first provides an overview of the allegations of discrimination made by the Assembly of First Nations and the First Nations Child and Family Caring Society in their human rights complaint against Canada regarding the jurisdictional disputes and lack of coordination between the various levels of government and between government departments and the harmful impact they have on First Nations children. It then summarizes the initial decision of the Canadian Human Rights Commission [CHRC] in January 2016 concerning Jordan's Principle. The problems and delays associated with implementation of the CHRC decision are examined, and the May 2017 CHRC order compelling Canada to take concrete measures to comply with its initial decision is reviewed in detail. The final part of the paper advances the position that in order to genuinely ensure substantive equality in Canadian society, Canada must be more proactive in identifying and remedying its discriminatory practices. In this case, putting the Spirit Bear Plan into effect within the Canadian government offers a

* $\quad$ B.A., LL.B., MSt (Oxon). Anne Levesque est professeure adjointe au Programme de common law français de la Faculté de droit de l'Université d'Ottawa. Avec David Taylor, Sarah Clarke, Barbara McIsaac et Sébastien Grammond (avant qu'il soit nommé juge à la Cour fédérale du Canada en novembre 2017), l'autrice est membre de l'équipe juridique qui représente la Société de soutien à l'enfance et à la famille des Premières Nations du Canada dans la plainte déposée conjointement avec l'Assemblée des Premières Nations, contre le gouvernement du Canada. 
promising way forward for ending injustices in the delivery of public services for First Nations children.

\section{INTRODUCTION}

En 2007, l'Assemblée des Premières Nations du Canada [APN] et la Société de soutien à l'enfance et à la famille des Premières Nations du Canada (Société de soutien) déposent une plainte de droits de la personne alléguant la discrimination raciale de la part du gouvernement du Canada dans la prestation de services d'aide à l'enfance à plus de 165000 enfants des Premières Nations ${ }^{1}$. L'une des allégations avancées dans la plainte est que le manque de coordination et les conflits de compétence au sein du gouvernement fédéral, entre ses ministères, ainsi qu'avec les différents paliers de gouvernements provinciaux, ont des conséquences importantes sur les enfants des Premières Nations. En effet, plusieurs d'entre eux doivent attendre de longues périodes de temps avant de recevoir les services dont ils ont désespérément besoin, ou reçoivent des services inéquitables, tandis que d'autres se voient refuser des services qui sont offerts aux autres enfants du Canada ${ }^{2}$. La plainte allègue que cette conjoncture constitue de la discrimination fondée sur la race et sur l'origine ethnique ou nationale, en contravention de l'article 5 de la Loi canadienne sur les droits de la personne [LCDP ou Loi] $]^{3}$. En janvier 2016, le Tribunal canadien des droits de la personne [TCDP ou Tribunal] conclut que les iniquités dans la conception et les formules de financement du système de services d'aide à l'enfance sont discriminatoires envers les enfants des Premières Nations et portent atteinte à la $\mathrm{LCDP}^{4}$. De surcroit, il accepte l'argument des plaignants selon lequel le manque de coordination entre les différents programmes entraine des interruptions, des retards ou des refus de services destinés aux enfants de Premières Nations. Selon le Tribunal, ceci constitue un acte discriminatoire au sens de l'article 5 de la Loi. Il ordonne donc au Canada de mettre en œuvre le principe de Jordan immédiatement et de façon intégrale ${ }^{5}$. Après plus d'un an de retards et de problèmes bien documentés dans la mise en œuvre du principe de Jordan au sein du gouvernement, le TCDP rend en mai 2017 une nouvelle ordonnance obligeant le Canada à prendre une série de mesures concrètes pour se conformer à sa décision initiale et pour assurer le respect du droit à l'égalité réelle des enfants des Premières Nations du Canada ${ }^{6}$.

1 Assemblée des Premières Nations et Société de soutien à l'enfance et à la famille des Premières Nations du Canada.

Plainte de droits de la personne contre le gouvernement du Canada, Ottawa (23 février 2007) [ci-après « La plainte »], en ligne : Société de soutien à l'enfance et à la famille des Premières Nations du Canada

$<$ https://fncaringsociety.com/sites/default/files/Caring\%20Society_AFN\%20HR\%20complaint\%202007.pdf $>$. Voir aussi Phil Fontaine, "Speaking Notes for Assembly of First Nations National Chief Phil Fontaine International Conference on Ethics" (12 février 2007), en ligne : NationalTalk $<$ http://nationtalk.ca/story/speaking-notes-for-assembly-of-firstnations-national-chief-phil-fontaine-international-conference-on-ethics $>$.

2 La plainte, $i b i d$.

3 Loi canadienne sur les droits de la personne, L.R.C., 1985, ch. H-6, art. 5.

4 Société de soutien à l'enfance et à la famille des Premières Nations du Canada et al. c. Procureur général du Canada (pour le ministre des Affaires indiennes et du Nord canadien), 2016 TCDP 2 [ci-après « décision sur le fond » ou « décision de janvier $2016 »]$ aux par. 341 à 382.

$5 \quad$ Ibid. au par. 481.

$6 \quad$ Société de soutien à l'enfance et à la famille des Premières Nations c. Procureur général du Canada (représentant le ministre des Affaires autochtones et du Nord canadien), 2017 TCDP 14 [ci-après « ordonnance de mai 2017 ». 
Le présent article porte sur les problèmes et les retards dans la mise en œuvre du principe de Jordan au sein du gouvernement du Canada dans cette cause historique. Il se décline en cinq parties. Premièrement, l'article passe en revue l'ensemble des allégations de discrimination mises de l'avant par l'APN et la Société de soutien dans leur plainte de droits de la personne en ce qui a trait aux conflits de compétence et du manque de coordination entre les différents paliers et ministères de gouvernements, et leurs répercussions néfastes sur les enfants des Premières Nations. Deuxièmement, il résume la décision initiale du TCDP, rendue en janvier 2016, en ce qui a trait au principe de Jordan. Troisièmement, l'article examine les problèmes et les retards liés à la mise en œuvre de la décision du TCDP. Quatrièmement, il aborde en détail l'ordonnance de mai 2017 du TCDP obligeant le Canada à prendre des mesures concrètes pour se conformer à sa décision initiale. Il examine aussi les autres ordonnances rendues par le Tribunal contre le Canada en ce qui a trait au principe de Jordan. Enfin, l'article propose la thèse suivante : afin d'assurer l'égalité réelle pour les enfants des Premières Nations, le Canada doit être plus proactif dans l'identification et la rectification de ses pratiques discriminatoires. En l'occurrence, la mise en œuvre du «Plan de l'Ourson Spirit » au sein du gouvernement canadien offre une voie prometteuse vers la fin des iniquités dans la prestation de services publics pour les enfants des Premières Nations.

\section{L'INIQUITÉ DES SERVICES GOUVERNEMENTAUX OFFERTS AUX ENFANTS DES PREMIÈRES NATIONS}

En février 2007, l'APN et la Société de soutien déposent une plainte de droits de la personne alléguant la discrimination raciale de la part du gouvernement du Canada dans sa prestation de services d'aide à l'enfance à plus de 165000 enfants des Premières Nations et à leurs familles'. L'une des allégations avancées dans la plainte est que le manque de coordination et les conflits de compétence au sein du gouvernement fédéral, entre ses ministères, ainsi qu'avec les différents paliers de gouvernements provinciaux, ont des conséquences importantes sur les enfants des Premières Nations. En effet, plusieurs d'entre eux doivent attendre de longues périodes de temps avant de recevoir les services dont ils ont désespérément besoin, ou reçoivent des services inéquitables, tandis que d'autres se voient refuser des services qui sont offerts aux autres enfants du Canada ${ }^{8}$.

Jordan River Anderson était l'un des nombreux enfants de Premières Nations qui se sont vu refuser des services gouvernementaux en raison d'un conflit de compétence entre différents paliers de gouvernements ${ }^{9}$. La famille de Jordan est membre de la Nation crie de Norway House au Manitoba ${ }^{10}$. En 1999, Jordan naît dans un hôpital de Winnipeg à plus de $400 \mathrm{~km}$ de sa communauté en raison des complications médicales survenues durant la grossesse de sa mère ${ }^{11}$. Il doit y rester après sa naissance

7 La plainte, supra note 2. La Commission canadienne des droits de la personne était une partie à l'audience qui, en vertu de l'article 51 de la Loi sur les droits de la personne supra note 3, était tenue d'adopter l'attitude la plus proche de l'intérêt public. Les Chiefs of Ontario et Amnistie internationale ont aussi participé à l'audience sur le fond à titre de parties intéressées. La Nation Nishnawbe-Aski a obtenu le statut de partie intéressée après que la décision sur le fond a été rendue. Voir Société de soutien à l'enfance et à la famille des Premières Nations du Canada et al. c. Procureur général du Canada (pour le ministre des Affaires indiennes et du Nord canadien), 2016 TCDP 11.

8 La plainte, ibid.

$9 \quad$ La décision sur le fond, supra note 5 au par. 352.

10 Ibid.

11 Ibid. 
pour recevoir des soins particuliers, car les services médicaux dont il a besoin ne sont pas disponibles sur la réserve où habite sa famille ${ }^{12}$. Après avoir été hospitalisé pendant les deux premières années de sa vie, Jordan reçoit enfin l'autorisation de son équipe médicale de rentrer chez lui ${ }^{13}$. Le bambin a toutefois besoin de certains services médicaux à domicile (qui sont couramment offerts par la province aux enfants vivant hors réserve) afin de faire la transition vers une vie normale à la maison ${ }^{14}$. Or, comme Jordan est un enfant des Premières Nations, le ministère des Affaires autochtones et Développement du Nord Canada $[\mathrm{AADNC}]^{15}$, Santé Canada et le gouvernement du Manitoba ne parviennent pas à s'entendre sur le palier ou le ministère gouvernemental qui devrait payer les services de Jordan après son congé de l'hôpital ${ }^{16}$. Parce qu'aucun palier de gouvernement n'accepte de fournir à Jordan les services dont il a besoin pour entamer le processus menant à son retour éventuel dans sa famille et dans sa communauté de Norway House, il est contraint de demeurer à l'hôpital à Winnipeg ${ }^{17}$. Entre-temps, AADNC, Santé Canada et la province du Manitoba continuent à se «renvoyer la balle pour savoir qui devrait financer les soins à domicile de Jordan. ${ }^{18}$ » Le jeune garçon décède à l'âge de cinq ans, après avoir passé toute sa vie à l'hôpital, malgré l'autorisation de sortir trois ans plus tôt. Au moment de sa mort, les fonctionnaires des gouvernements du Canada et du Manitoba se disputaient encore par rapport à qui devait payer les services qui auraient permis à Jordan de quitter l'hôpital ${ }^{19}$.

L'expérience tragique de Jordan et de sa famille n'est pas un cas isolé. En fait, de nombreux rapports révèlent que les enfants et les familles des Premières Nations qui sollicitent des services gouvernementaux se heurtent fréquemment à de tels bourbiers bureaucratiques. Par exemple, une étude menée en 2005 auprès de douze agences de protection de l'enfance des Premières Nations à travers le Canada, relève près de 400 conflits de compétence au cours d'une seule année, causant des retards et même des refus de services gouvernementaux aux enfants des Premières Nations ${ }^{20}$. Un rapport de 2008 de la vérificatrice générale du Canada sonne également l'alarme par rapport à l'ampleur de ce problème et documente ses conséquences néfastes sur les enfants des Premières Nations, dont notamment leur déracinement familial et le placement dans des foyers d'accueil21. Selon le rapport:

Nous avons constaté que les organismes des Premières nations ne peuvent pas toujours compter sur d'autres services sociaux et de santé pour les aider à garder des familles unies ou à offrir les services nécessaires aux Premières nations. L'accès à ces services n'est pas

12 Ibid.

13 Ibid.

14 Ibid.

15 Lors de la rédaction de la plainte, l'intimé se nommait ministre des Affaires indiennes et du Nord Canada. Lors de l'audience, l'intimé se nommait ministère des Affaires autochtones et Développement du Nord («AADNC»). Voir la décision sur le fond, supra note 5 au par. 5.

16 La décision sur le fond, supra note 5 au par. 352.

17 Ibid.

18 Ibid.

19 Ibid.

20 Cindy Blackstock et coll., Wen: De, Nous voyons poindre la lumière du jour (Ottawa, Société de soutien à l'enfance et à la famille des Premières Nations, 2005).

21 Vérificatrice générale du Canada, Rapport de mai 2008 de la vérificatrice générale du Canada à la Chambre des communes, chapitre 4, Programme des services à l'enfance et à la famille des Premières Nations - Affaires indiennes et du Nord Canada (Ottawa, ministre des Travaux publics et des Services gouvernementaux Canada, 2008). 
le même dans les collectivités situées à l'intérieur et à l'extérieur des réserves, et il diffère également d'une Première nation à l'autre. [...] Les enfants des Premières nations qui ont besoin d'importants soins médicaux se trouvent dans une situation ambigüe ${ }^{22}$.

En février 2007, l'APN et la Société de soutien déposent une plainte de droits de la personne contre le Canada à la Commission canadienne des droits de la personne ${ }^{23}$. La plainte allègue que le manque de coordination et les conflits de compétence, comme celui ayant mené au refus de services à Jordan Anderson, ont des effets néfastes sur les enfants des Premières Nations et constituent de la discrimination fondée sur la race et l'origine ethnique ou nationale en contravention de l'article 5 de la Loi canadienne sur les droits de la personne ${ }^{24}$. Les plaignants implorent le Tribunal d'ordonner au gouvernement du Canada de mettre en œuvre immédiatement et intégralement le principe de Jordan élaboré en hommage à Jordan River Anderson. Il s'agit d'un principe axé sur les besoins réels des enfants et qui, à l'époque, se définissait comme suit :

[U]n principe qui place l'intérêt de l'enfant en premier. Il prévoit que lorsqu'un service gouvernemental est offert à tous les autres enfants et qu'un conflit de compétences surgit entre le Canada et une province ou un territoire, ou entre des ministères du même gouvernement, au sujet de services offerts à l'enfant d'une Première Nation, le ministère contacté en premier doit payer pour les services. Il peut demander à être remboursé par l'autre ministère ou gouvernement, après que l'enfant ait reçu le service. Ce principe vise à empêcher que les enfants des Premières Nations se voient refuser des services publics essentiels ou qu'ils soient laissés en attente de services ${ }^{25}$.

Après des années de tactiques procédurales infructueuses par les avocats du gouvernement du Canada tentant de faire rejeter la plainte de façon préliminaire, coûtant des milliers de dollars aux contribuables canadiens $^{26}$, le Tribunal amorce enfin l'audience sur le fond en février $2013^{27}$. Au cours de l'audience de 72 jours, le Tribunal entend 25 témoins et examine plus de 500 documents déposés en preuve ${ }^{28}$. C'est après cette audience, suivie d'un an et demi de délibérations, que le Tribunal rend sa décision historique

Ibid. à la page 13.

La plainte, supra note 2 .

Ibid.

La décision sur le fond, supra note 5 au par. 351.

26 En 2016, le gouvernement fédéral avait dépensé au moins 12 millions de dollars en frais juridiques liés à cette plainte. Voir Broadbent Institute, "The Federal Government Picked a Fights with First Nations Kids. Three Years and Millions in Legal Fees Later, They Lost" (6 septembre 2019), en ligne : Press Progress $<$ https://pressprogress.ca/the-federal-governmentpicked-a-fight-with-first-nations-kids-three-years-and-millions-in-legal-fees-later-they-lost/>.

27 Pour un compte rendu complet de l'histoire de la procédure de la cause, voir Cindy Blackstock, "The Complainant: The Canadian Human Rights Case on First Nations Child Welfare" (2016) 62: 2 McGill LJ 285-328. Voir Anne Levesque, Sarah Clarke et Cindy Blackstock, « La plainte de discrimination devant le Tribunal canadien des droits de la personne portant sur les services d'aide à l'enfance aux enfants des Premières Nations et le Principe de Jordan » (2016) 25 Enfance, Famille, Générations 1-18.

28 La décision sur le fond, supra note 5 aux par. 7-10 et 14. 
sur les allégations de discrimination, y compris le manquement quant à la mise en œuvre du principe de $\operatorname{Jordan}^{29}$.

\section{LA VICTOIRE DES ENFANTS DES PREMIÈRES NATIONS}

La décision du TCDP, rendue en janvier 2016, est une victoire absolue pour les enfants des Premières Nations. Sous la plume de ses deux membres, la formation du TCDP accepte de manière non équivoque toutes les allégations de discrimination mises de l'avant par les plaignants ${ }^{30}$. Le Tribunal conclut notamment que les conflits de compétence et le manque de coordination dans les services publics offerts aux enfants des Premières Nations ont un impact discriminatoire et portent atteinte à l'article 5 de la $\mathrm{LCDP}^{31}$. Plusieurs sont d'avis que le soutien de plusieurs milliers de personnes qui ont fait preuve de solidarité envers les enfants des Premières Nations lors de l'audience a joué un rôle important dans cette victoire $^{32}$. En effet, pendant que se déroulait l'audience, une campagne populaire faisait boule de neige. Connue sous le nom de « Je suis un témoin », la campagne encourage « les gens à apprendre au sujet du cas sur la protection de l'enfance des Premières Nations ainsi que le Principe de Jordan ».

La première phrase de la décision donne le ton : «La présente décision concerne les enfants », souligne le Tribunal ${ }^{33}$. Cette préoccupation réelle pour l'intérêt supérieur de l'enfant imprègne toute la décision du Tribunal qui décrit méticuleusement l'effet des politiques discriminatoires du Canada sur les victimes et leurs familles. Après avoir abordé en détail les allégations des plaignants en ce qui a trait au financement inéquitable des services d'aide à l'enfance offerts aux enfants des Premières Nations, le Tribunal fait le bilan des démarches prises par le gouvernement du Canada visant à mettre en œuvre le principe de $J_{0}$ ordan $^{34}$. Il reconnait que Santé Canada et AADNC avaient élaboré un protocole d'entente à cet effet en 2009. Fondée sur un principe de collaboration interministérielle ${ }^{35}$, la première version du protocole vise à «assurer que les différends en matière de financement n'interrompent pas les services offerts aux

29 Ibid. aux par. 341 à 382.

30 Un résumé des conclusions de fait est fourni supra note 5 au par. 458. Pour un résumé détaillé de la décision, voir aussi Anne Levesque. «La victoire des enfants de Premières Nations et leur lutte continue pour parvenir à l'égalité réelle » (2016/2018) Vol II, Annuaire canadien des droits de la personne, 39. Une formation de trois membres était initialement constituée pour entendre la plainte; la présidente Sophie Marchildon, le membre Réjean Bélanger et le membre Edward Lustig. Au cours des délibérations, le membre Bélanger est décédé. Pour cette raison, seuls la présidente Marchildon et le membre Lustig ont signé la décision. L'autrice salue la précieuse contribution du membre Bélanger durant l'audience de cette cause. Voir supra note 5 aux par. 5 à 9.

31 Voir Société de soutien à l'enfance et aux familles des Premières Nations du Canada, « Je suis un témoin » (6 septembre 2019) en ligne : <https://fncaringsociety.com/fr/je-suis-un-témoin>. Pour une étude approfondie du mouvement social qui a appuyé le litige, voir Anne Levesque, "The Symbiotic Relationship Between Social Movements and Public Interest Litigation : A Case Study of the I am a Witness Campaign and the Human Rights Complaint of 165,000 First Nations Kids" 90 : 2 SCLR 101-106. L'audience était la première au Canada à être télévisée grâce à une décision de la Cour fédérale du Canada dans Réseau de télévision des peuples autochtones c. Canada (Commission des droits de la personne), $2011 \mathrm{CF} 810$. Le litige et le mouvement social connexe ont aussi fait l'objet de deux documentaires de l'éminente réalisatrice autochtone Alanis Obomsawin. Voir Office national du film du Canada, « Alanis Obomsawin » en ligne : <https://www.onf.ca/cineastes/alanis-obomsawin/>

32 Voir Blackstock, supra note 27 au par. 59 et Levesque Ibid. à la page 106.

33 La décision sur le fond, supra note 4 au par. 1.

34 Ibid. par. 341 à 382.

35 Ibid. au par. 354 
enfants $»^{36}$. Le protocole est renouvelé en 2013, sans modification importante sur le fond ${ }^{37}$. Le TCDP constate des lacunes importantes dans la portée du principe de Jordan tel que défini dans le protocole ainsi que dans sa mise en œuvre. Plus précisément, le protocole s'appliquait uniquement aux demandes mettant en cause un conflit entre un gouvernement provincial et le gouvernement du Canada et entre les ministères fédéraux, alors que cela ne représente que 14 pour cent des demandes reçues en vertu du principe de Jordan $^{38}$. De plus, seuls les enfants ayant de multiples handicaps étaient admissibles. Finalement, les services en question devaient être offerts aux enfants résidant à l'extérieur de la réserve, dans la même région afin de satisfaire aux critères du protocole ${ }^{39}$. Selon le TCDP, ces critères de recevabilité avaient pour effet de priver les enfants des Premières Nations de services gouvernementaux dont ils avaient besoin ${ }^{40}$. D'ailleurs, la bureaucratie complexe entourant le principe de Jordan, tout comme l'application de critères étroits et incohérents lors des prises de décisions sur les demandes de services, ont fait en sorte qu'il était tout à fait impossible d'accéder au financement du gouvernement du Canada visant à réduire les iniquités des services offerts aux enfants des Premières Nations ${ }^{41}$. Comme le constate le TCDP, le fonds de 11 millions de dollars attribués par Santé Canada pour traiter des demandes fondées sur le principe de Jordan n'ayant jamais été utilisé dans ses trois années d'existence a donc été abandonné ${ }^{42}$.

Le TCDP se penche ensuite sur les effets néfastes des conflits de compétence et du manque de coordination dans les services publics pour les enfants des Premières Nations. Selon le Tribunal, ceci se « traduit par des interruptions, des délais et des refus de services pour les enfants des Premières Nations vivant dans les réserves $»^{43}$. À titre d'exemple, il évoque le cas d'une enfant de quatre ans d'une Première Nation qui subit un arrêt cardiaque ainsi qu'une lésion cérébrale anoxique lors d'un examen dentaire de routine ${ }^{44}$. La jeune fille devient complètement dépendante d'un soignant pour toutes ses activités quotidiennes ${ }^{45}$. Afin de recevoir son congé de l'hôpital, elle a besoin d'accès à un équipement médical spécialisé à domicile, dont une poussette adaptée, un lit, un matelas, un lève-personne et un système de rails au plafond ${ }^{46}$. Une demande de financement est faite auprès de Santé Canada, mais celle-ci est refusée $^{47}$. De même, la province, AADNC et l'agence régionale de santé refusent aussi de financer le matelas et le lit pour la fillette ${ }^{48}$. De ce fait, aucun palier de gouvernement ou ministère gouvernemental n'accepte de financer ou de rembourser l'équipement ${ }^{49}$. Même dans ces circonstances, Santé Canada continue de refuser catégoriquement la demande. Après plusieurs mois d'attente, la fillette reçoit l'équipement médical dont elle a besoin grâce à un don privé fait de façon anonyme ${ }^{50}$. N'eût été ce geste

\footnotetext{
Ibid. au par. 357

Ibid. au par. 358

Ibid.

Ibid. au par. 359.

Ibid. aux par. 379-382.

Ibid. aux par. 380-381.

Ibid. au par. 380 .

Ibid. au par. 381

Ibid. au par. 366.

Ibid.

Ibid.

Ibid.

Ibid. au par. 367.

Ibid.

Ibid. aux par. 366 et 367
} 
de charité, la jeune fille n'aurait jamais eu accès à l'équipement dont elle avait besoin ${ }^{51}$. Dans le même ordre d'idées, le TCDP constate que le manque de coordination et les conflits de compétence sont particulièrement graves et fréquents dans la prestation de services de soins de santé pour les enfants pris en charge par l'État, de services de santé mentale et de soins dentaires ${ }^{52}$. Finalement, le Tribunal souligne que l'effet discriminatoire de la définition étroite du principe de Jordan a déjà fait l'objet d'un litige devant la Cour fédérale du Canada dans l'affaire Conseil de la bande de Pictou Landing c. Canada (Procureur général $)^{53}$. Dans cette décision concernant une demande de financement pour des soins à domicile pour un jeune garçon atteint d'hydrocéphalie, d'infirmité motrice cérébrale, de courbure rachidienne et d'autisme, la Cour conclut que la définition du Canada du principe de Jordan est trop étroite et donc déraisonnable ${ }^{54}$.

À la lumière de ses conclusions de fait, le Tribunal statue que

L'absence de coordination du Programme des SEFPN et des autres ententes provinciales/territoriales connexes avec d'autres ministères et d'autres programmes et services du gouvernement destinés aux Premières Nations dans les réserves, a entraîné des interruptions, des retards et des refus de services pour les enfants des Premières Nations ${ }^{55}$.

Le Tribunal se penche aussi sur l'allégation des plaignants selon laquelle les manquements dans la mise en œuvre pleine et complète du principe de Jordan sont discriminatoires et contraires à la Loi. Il accepte l'argument et conclut que « la définition étroite et l'application insuffisante du principe de Jordan, ont entraîné des interruptions, des retards et des refus de services pour les enfants des Premières Nations $»^{56}$. Par conséquent, il ordonne au Canada « de cesser d'appliquer sa définition étroite du principe de Jordan et de prendre des mesures pour appliquer immédiatement le principe de Jordan en lui donnant sa pleine portée et tout son sens ${ }^{57}$. De plus, le TCDP implore le Canada d'agir dans l'intérêt supérieur des enfants pour qu'ils aient «droit à l'égalité des chances d'épanouissement et à la prise de mesures visant à la satisfaction de leurs besoins » comme le prévoit la $\operatorname{LCDP}^{58}$. Le Tribunal conclut en avisant les parties qu'il communiquera avec elles dans les semaines suivant la décision sur le fond afin de mettre au point un processus pour aborder les questions demeurant en suspens en ce qui a trait aux mesures de réparation demandées par les parties ${ }^{59}$.

$51 \quad$ Ibid. au par. 367.

52 Ibid. aux par. 370-372.

53 Ibid. au par. 376. Conseil de la bande de Pictou Landing c. Canada (Procureur général), 2013 CF 342.

54 La décision sur le fond, supra note 4 au par. 378.

$55 \quad$ Ibid. au par. 458.

56 Ibid. au par. 458.

57 Ibid. aux par. 351 et 481.

58 Ibid. au par. 482.

59 Ibid. au par. 484. 


\section{LE CANADA FAIT FI DE L'ORDONNANCE DU TCDP}

En rétrospective, la conclusion du Tribunal affirmant que le gouvernement du Canada était au courant depuis de nombreuses années des répercussions néfastes de son comportement discriminatoire sur les enfants des Premières Nations, sans pour autant agir, préfigurait son inaptitude à se soumettre à l'ordonnance exigeant la mise en œuvre immédiate et intégrale du principe de Jordan ${ }^{60}$. Lorsque le Tribunal demande un rapport d'étape sur la mise en œuvre du principe de Jordan après avoir rendu sa décision sur le fond en janvier 2016, le Canada est incapable de fournir d'explications précises quant aux mesures concrètes prises pour se conformer à l'ordonnance. Bien que le Canada ait droit à deux tentatives de s'expliquer au Tribunal et aux parties, le manque de précision par rapport à son plan pour mettre en œuvre la décision du Tribunal est étonnant. Dans son premier rapport au Tribunal, le Canada envisage de commencer des discussions élargies concernant le principe de Jordan ${ }^{61}$. Déployant manifestement de grands efforts pour éviter l'utilisation du terme " consultation », le Canada propose plutôt un processus d'engagement avec les partenaires en vue d'élargir la définition du principe et d'améliorer sa mise en œuvre ${ }^{62}$. Sans vouloir « prédéterminer » le travail qui reste à faire ou l'échéancier à respecter, il suggère que des options pourraient être élaborées au cours des douze prochains mois ${ }^{63}$. Lorsque les parties dénoncent l'inaction du Canada à l'égard de la décision dans leurs mémoires au Tribunal, le Canada dépose une réplique qui est encore davantage truffée d'ambigüités. D'abord, le Canada explique qu'il avait amorcé des « discussions internes » visant à élargir la définition du principe de Jordan et avait commencé à identifier des partenaires qui seraient impliqués dans le processus de sa mise en œuvre ${ }^{64}$. Ensuite, il prétend qu'il prévoyait d'entamer d'autres discussions avec les provinces, les territoires et les Premières Nations au cours des prochains mois et que des options pour modifier le principe de Jordan seraient élaborées dans douze mois ${ }^{65}$. Le Canada ne dépose aucune preuve au Tribunal qui permette d'établir l'existence de ces discussions internes, d'un plan de travail ou d'un bilan, même préliminaire, des partenaires ciblés par ledit processus d'engagement.

Face au manque de précision et d'action concrète de la part du Canada, le Tribunal rend une deuxième décision en avril 2016. Il rappelle que son ordonnance de janvier 2016 obligeant le Canada à mettre en œuvre le principe de Jordan doit être respectée intégralement et immédiatement ${ }^{66}$. Il ordonne à nouveau au Canada de mettre en œuvre sans délai le principe de Jordan de manière à inclure tous les conflits de

60 Ibid. aux par. 462 à 463.

61 Gouvernement du Canada, "Respondent's Submissions on Remedy" (10 mars 2016), en ligne : Société de soutien à l'enfance et aux familles des Premières Nations du Canada

$<$ https://fncaringsociety.com/sites/default/files/Federal\%20government\%20submissions\%20on\%20immediate\%20relief. pdfs

$62 \quad$ Ibid. au par. 2.

63 Ibid. au par. 27.

64 Gouvernement du Canada, "Respondent's FurtherSubmissions on Remedy" (6 avril 2016), en ligne : Société de soutien à l'enfance et aux familles des Premières Nations du Canada $<$ https://fncaringsociety.com/sites/default/files/Respondent $\% 27 \mathrm{~s} \% 20$ Further\%20Submissions\%20on\%20Remedy $\% 20$ $\% 20$ April\%206\%2C\%202016.pdf >

65 Société de soutien à l'enfance et à la famille des Premières Nations du Canada et al. c. Procureur général du Canada (pour le ministre des Affaires indiennes et du Nord canadien), 2016 TCDP 10 au par. 31.

$66 \quad$ Ibid. au par. 32. 
compétence, y compris les différends entre ministères fédéraux et à l'égard de tous les enfants des Premières Nations (non seulement ceux qui ont plusieurs handicaps ${ }^{67}$. Plus concrètement, il réitère que « l'organisme gouvernemental qui est contacté en premier devrait payer le service sans devoir procéder à un examen au regard des politiques ni tenir des conférences sur le cas avant qu'un financement soit fourni. ${ }^{68}$ » De plus, il implore au Canada de se conformer à « l'esprit et à l'objet du principe de Jordan » et de respecter l'intérêt supérieur de l'enfant ${ }^{69}$. Finalement, le Tribunal demande au Canada de lui fournir un autre compte rendu sur son progrès dans les deux semaines de sa décision ${ }^{70}$.

Le rapport déposé par le Canada en réponse à l'ordonnance du TCDP d'avril 2016 continue à décevoir. Dans sa réponse datée du 6 juillet 2016, le Canada défend ladite «nouvelle approche » au principe de Jordan. Étonnamment, cette approche est fondée sur une définition du principe que le Tribunal avait jugé discriminatoire dans sa décision de janvier 2016. Plus précisément, le rapport révèle que le Canada accepte uniquement les demandes de services pour les enfants des Premières Nations vivant dans les réserves, ayant un handicap ou une condition médicale à court terme et nécessitant des services sociaux ou de santé $^{71}$. Le Canada prétend toutefois adopter « le principe de l'enfant d'abord», un terme qui est de toute évidence vide de sens étant donné sa définition étroite du principe qui n'a permis à aucun enfant d'accéder aux services dont il avait besoin.

À la réception de ce deuxième rapport d'étape du Canada, le Tribunal rend une troisième ordonnance en septembre $2016^{72}$. Dans cette décision, il constate encore une fois que l'intimé interprète toujours le principe de Jordan étroitement et de manière qui «ne correspond pas avec la décision » ${ }^{73}$. Le TCDP souligne que le principe ne s'applique pas uniquement aux enfants des Premières Nations vivant dans les réserves, mais à tous les enfants des Premières Nations ${ }^{74}$. Il exprime également des préoccupations par rapport à la formulation du principe de Jordan adoptée par le gouvernement du Canada qui se limite aux enfants « atteints de problèmes de santé et ceux qui présentent un problème spécifique à court terme qui nécessite une aide sociale et médicale. ${ }^{75}$ » Selon le Tribunal, cette interprétation étroite risque d'entraîner des restrictions dans les services offerts aux enfants des Premières Nations et pourrait avoir un effet discriminatoire $^{76}$. Il ordonne ainsi au Canada de lui fournir d'autres précisions sur les différents aspects de la définition, mise en œuvre du principe de Jordan et il organise une conférence de gestion des cas pour en discuter ${ }^{77}$.

67 Ibid. au par. 33.

68 Ibid. au par. 33.

69 Ibid. au par. 33.

$70 \quad$ Ibid. au par. 34.

71 Gouvernement du Canada, Respondent's Further Reply Submissions Re Immediate Relief (6 juillet 2016) au par. 34, en ligne : Société de soutien à l'enfance et aux familles des Premières Nations du Canada $<$

https://fncaringsociety.com/sites/default/files/Federal\%20government\%20compliance\%20report_July\%206\%2C\%20201 6.pdf>.

72 Ibid.

73 Société de soutien à l'enfance et à la famille des Premières Nations du Canada et autres c. Procureur général du Canada (représentant le ministre des Affaires autochtones et du Nord canadien), 2016 TCDP 16 (CanLII)

$74 \quad$ Ibid. au par. 117.

75 Ibid. au par. 119.

76 Ibid. aux par. 117 et 118 .

77 Ibid. aux par. 119. 
Confrontés à un intimé qui s'est révélé soit incapable ou peu disposé à se conformer à trois décisions contraignantes, les plaignants et les parties intéressées déposent des requêtes demandant au Tribunal de déclarer que le Canada ne respecte toujours pas ses ordonnances visant à assurer la mise en œuvre immédiate et intégrale du principe de Jordan ${ }^{78}$. Ils implorent le TCDP d'ordonner d'autres mesures réparatrices concrètes pour assurer l'égalité réelle dans la prestation de services gouvernementaux offerts aux enfants des Premières Nations ${ }^{79}$.

Peu après que le processus pour entendre les requêtes est amorcé, les retards dans la mise en œuvre pleine et entière du principe de Jordan entrainent des conséquences mortelles lorsque deux enfants de douze ans de la Première Nation Wapekeka au nord de l'Ontario meurent par suicide ${ }^{80}$. Même le Canada ne conteste pas la preuve irréfutable que ces morts tragiques auraient pu être évitées. En effet, la Nation Wapekeka, en apprenant qu'il y avait eu un pacte de suicide parmi des jeunes de la communauté quelques mois avant l'incident, avait fait une proposition détaillée à Santé Canada afin de prévenir ce dénouement tragique $^{81}$. Cependant, la demande de financement est ignorée par Santé Canada qui déplore qu'elle soit venue à un « moment peu propice dans le cycle de financement fédéral. ${ }^{82}$. Ce n'est seulement qu'après la mort de ces enfants que Santé Canada révise et accepte enfin la demande de financement ${ }^{83}$. L'horrible leçon à tirer de cette situation est que les enfants et les jeunes des Premières Nations ont perdu toute attente de recevoir des services équitables ${ }^{84}$.

\section{L'ORDONNANCE CONTRE LE CANADA}

En mai 2017, le Tribunal rend sa décision relative aux requêtes des plaignants et des parties intéressées concernant le manquement du Canada à mettre en œuvre le principe de Jordan ${ }^{85}$. Comme dans toutes ses décisions antérieures liées à cette plainte, le Tribunal accepte tous les arguments mis de l'avant par les plaignants et les parties intéressées ${ }^{86}$. En particulier, les membres de la formation constatent que, malgré sa décision de janvier 2016, «[1]e Canada a continué d'agir de la même manière et a conservé son approche étroite à l'égard du principe de Jordan. ${ }^{87}$ » Le Tribunal ajoute que le Canada était conscient qu'il ne respectait pas sa décision prise en janvier 2016. Il décrit l'approche du Canada comme étant une :

78 Société de soutien à l'enfance et aux familles des Premières Nations du Canada, Requête au TDCP sur le défaut du Canada de se conformer aux ordonnances du Tribunal concernant le principe de Jordan (16 décembre 2016) en ligne : Société de soutien à l'enfance et aux familles des Premières Nations du Canada

$<$ https://fncaringsociety.com/sites/default/files/fncfcsc_submission_re_jordans_principle_-_december_16_2016.pdf >

79 Ibid.

80 Société de soutien à l'enfance et à la famille des Premières Nations c. Procureur général du Canada (représentant le ministre des Affaires autochtones et du Nord canadien), 2017 TCDP 14. L'autrice offre ses plus sincères condoléances aux familles et aux membres de la communauté des victimes.

$81 \quad$ Ibid. au par. 88.

$82 \quad$ Ibid. au par. 89.

83 Ibid. au par. 89-90.

84 Nishnawbe Aski Nation, Mémoire sur les mesures de redressement immédiates (28 février 2017) en ligne : Société de soutien à l'enfance et aux familles des Premières Nations du Canada

$<$ https://fncaringsociety.com/sites/default/files/nan_immediate_relief_factum_february_28_2017.pdf >

85 L'ordonnance de mai 2017, supra note 7.

86 Ibid. au par. 80.

87 Ibid. au par. 50. 
décision stratégique calculée et éclairée, qui a été soumise à une analyse des répercussions financières et des risques potentiels, plutôt que d'être fondée sur les besoins ou l'intérêt supérieur des enfants des Premières Nations qui sont censés être protégés par le principe de Jordan et qui devraient être au cœur des programmes du Canada ${ }^{88}$.

En fait, le Tribunal constate que le Canada avait examiné quatre définitions du principe de Jordan et a sélectionné celle ayant la portée la plus étroite ${ }^{89}$. Ce faisant, l'intimé a reconnu que la définition qu'il a choisie pourrait faire l'objet de critiques en raison de l'accent mis sur certains enfants (à l'exclusion des autres) ainsi qu'en présentant d'autres lacunes potentielles ${ }^{90}$. Réitérant les motifs de sa décision de janvier 2016, le TCDP conclut que le Canada n'a pas complètement pris en compte les conclusions de fait et n'a pas suffisamment donné suite à ses ordonnances antérieures ${ }^{91}$. Il exprime également des préoccupations concernant le fait que le Canada continue à insister sur la comparabilité des services, contrairement à l'ordonnance du TCDP qui l'obligeait à offrir des services respectant l'égalité réelle des enfants des Premières Nations. Selon le Tribunal, les soins et les services disponibles hors réserve ne devraient pas forcément être utilisés pour établir la nature ou l'étendue des soins ou des services offerts aux enfants des Premières Nations ${ }^{92}$. Il résume ses conclusions comme suit :

L’interprétation étroite que fait le Canada du principe de Jordan, le manque de coordination entre ses programmes axés sur les enfants et les familles des Premières Nations (comme on le verra dans la prochaine section), l'accent qui est mis sur les politiques existantes et le fait d'éviter les coûts potentiellement élevés des services n'est pas l'approche appropriée pour mettre un terme à la discrimination. Au contraire, les décisions doivent être prises dans l'intérêt supérieur des enfants. Bien que les ministres de la Santé et des Affaires autochtones se soient engagés à soutenir l'intérêt supérieur des enfants, les renseignements qui proviennent de Santé Canada et d'AANC, comme le souligne la présente décision, ne cadrent pas avec ce qu'ont affirmé les ministres ${ }^{93}$.

En se fondant sur ces conclusions de fait, le Tribunal rend à nouveau une série d'ordonnances visant à assurer la mise en œuvre immédiate et intégrale du principe de Jordan et à éviter d'autres incidents de discrimination envers les enfants des Premières Nations. Ces ordonnances seront décrites ci-dessous.

\section{A. Modification de la définition du principe de Jordan}

Le Tribunal réitère que le Canada doit immédiatement cesser d'utiliser une définition étroite du principe de Jordan qui exclut, de façon injustifiée et illégale, certains groupes d'enfants de Premières Nations ${ }^{94}$. Il précise d'autant plus que le Canada doit adopter une définition qui protège l'intérêt supérieur

\footnotetext{
Ibid. au par. 55.

Ibid. au par. 52.

Ibid. au par. 50.

Ibid. au par. 67.

Ibid. au par. 53

Ibid. au par. 74.

Ibid. au par. 135(1)(A).
} 
de l'enfant ${ }^{95}$, qui vise à répondre aux besoins culturels des enfants des Premières Nations et qui assure leur égalité réelle ${ }^{96}$. Il ajoute que l'application du principe de Jordan ne devrait pas se limiter aux enfants habitant dans les réserves ${ }^{97}$ ou à ceux ayant une déficience ou une condition médicale ${ }^{98}$, ni seulement aux services sociaux et de santé ${ }^{99}$. De même, il précise que même si le principe de Jordan peut s'appliquer aux conflits de compétence qui surgissent au sein des gouvernements, un conflit n'est pas une condition nécessaire à son application ${ }^{100}$.

Le Tribunal ordonne au Canada d'appliquer cette définition élargie de façon immédiate ${ }^{101}$. Il interdit la diffusion de toute autre définition ${ }^{102}$. De plus, il ordonne au Canada de réexaminer toutes les demandes de services reçues depuis avril 2009 en utilisant cette nouvelle définition, et de revoir les ententes conclues avec des organismes de prestations de services pour assurer leur conformité avec l'ordonnance ${ }^{103}$. Il ordonne au Canada de terminer cette révision en cinq mois ${ }^{104}$.

\section{B. Modification des pratiques dans le traitement de demandes}

En plus d'ordonner au Canada d'appliquer une définition du principe de Jordan qui répond à de nombreux critères précis, le Tribunal prescrit le processus de traitement de demandes liées au principe de Jordan que le Canada doit mettre en œuvre. Plus particulièrement, il ordonne au Canada de traiter les demandes liées au principe de Jordan dans un délai de 12 heures lorsqu'il s'agit d'un cas urgent, et dans un délai de 48 heures pour tout autre cas ${ }^{105}$. Il précise que les procédures administratives, c'est-à-dire les discussions intragouvernementales ou intergouvernementales portant sur la prestation de services, ne doivent pas retarder l'offre de services à un enfant d'une Première Nation ${ }^{106}$. Le Tribunal enjoint au Canada de mettre en œuvre un nouveau système de traitement de demandes dans le prochain mois ${ }^{107}$.

95 Ibid. au par. 135 (1)B(i).

96 Ibid. au par. 135(1)(B)(iv).

97 Ibid. au par. 135 (1)B(i).

98 Ibid.

99 Ibid.

100 Ibid. au par. 135 (1)B(v).

101 Ibid. au par. 135(1)(A) et 135(1)(C).

102 Ibid.

103 Ibid. au par. 135(1)(D).

104 Ibid.

105 Le 23 juin 2017, le Canada a présenté une demande de contrôle judiciaire contestant certains aspects de l'ordonnance de mai 2017. Dans sa demande, il cherchait à obtenir l'annulation des paragraphes qui lui interdisaient de tenir des conférences sur la gestion de cas et l'obligeaient à faire l'évaluation initiale et à rendre une décision dans un délai de 12 à 48 heures suivant la réception de la demande. Après des discussions, les parties sont parvenues à un accord visant à modifier certains aspects de l'ordonnance de mai 2017 contestés dans la demande de contrôle judiciaire. Voir Société de soutien à l'enfance et à la famille des Premières Nations c. Procureur général du Canada (représentant le ministre des Affaires autochtones et du Nord canadien), 2017 TCDP 35 (CanLII). La version modifiée de l'ordonnance est disponible en anglais en ligne :

Société de soutien à l'enfance et aux familles des Premières Nations du Canada $<$ https://fncaringsociety.com/sites/default/files/2017_chrt_14_amended.pdf >

106 Supra note 7 au par. 135(2)(A)(iii).

107 Ibid. au par. 135(2)B. 


\section{Sensibilisation et formation}

Le Tribunal ordonne aussi au Canada de prendre une série de mesures concrètes visant à faire connaître, notamment aux fonctionnaires fédéraux et aux personnes étant les plus susceptibles d'avoir besoin de services, la portée élargie du principe de Jordan et le processus pour faire des demandes de services ${ }^{108}$. Tout d'abord, il ordonne au Canada d'afficher une nouvelle définition du principe de Jordan de manière «très claire et visible» sur son site Web. De plus, le Canada doit faire des annonces bilingues sur le Réseau de télévision des peuples autochtones du Canada expliquant la portée élargie du principe et le processus de demandes ${ }^{109}$. Il ordonne aussi au Canada de communiquer avec tous les intervenants qui ont reçu des documents au sujet du principe de Jordan depuis janvier 2016, ainsi que les fonctionnaires impliqués dans sa mise en œuvre, afin de les informer de la nouvelle définition et de leur expliquer l'ordonnance de mai $2017^{110}$. Le Tribunal précise que toutes les mesures de formation et de sensibilisation au sujet du principe de Jordan doivent être élaborées en consultation avec les plaignants et les parties intéressées ${ }^{11}$.

\section{Collection de données et production de rapport}

Finalement, le Tribunal ordonne au Canada de mettre en œuvre un système de repérage de traitement de demandes. Entre autres, le Canada doit tenir compte du nombre de demandes qu'il reçoit liées au principe de Jordan ainsi que les demandes de services qui pourraient être considérées de cette manière, les modalités de chaque demande, ses délais de traitement et le résultat de la demande ${ }^{112}$. Le TCDP ajoute que le Canada doit fournir un rapport et des affidavits qui décrivent en détail les mesures prises pour se conformer à ses autres ordonnances tous les six mois ${ }^{113}$. Il précise que les plaignants et les parties intéressées pourront fournir des réponses écrites aux rapports déposés par le Canada, contre-interroger les déposants et demander des ordonnances supplémentaires au besoin ${ }^{114}$.

\section{AU-DELÀ D’UNE PLAINTE DIRIGÉE PAR LES MODÈLES DE L'ÉGALITÉ}

La question qui subsiste est de savoir si d'autres ordonnances seront nécessaires pour veiller à ce que le gouvernement du Canada offre de manière cohérente et systématique des services qui tiennent compte des circonstances géographiques, culturelles et historiques des enfants des Premières Nations. Le principe de Jordan a été créé pour combler les lacunes et remédier aux iniquités systémiques dans la prestation des services fournis par les gouvernements aux enfants de Premières Nations. Pour l'instant, il s'agit d'un mécanisme $a d$ hoc, quoique nécessaire et salutaire, pour remédier aux programmes du gouvernement du Canada qui ne garantissent pas de manière systématique la prestation de services spécifiquement conçus

\footnotetext{
108 Ibid. au par. 135(3).

109 Ibid. au par. 135(3)(B).

110 Ibid. au par. 135(3)(C).

111 Ibid. au par. 135(E).

112 Ibid. au par. 135(2)(C).

113 Ibid. au par. 135(2)(D).

114 Ibid. au par. 135(4).
} 
pour répondre aux besoins géographiques, culturels et historiques des enfants des Premières Nations ${ }^{115}$. Cependant, le fardeau d'invoquer le principe de Jordan et de revendiquer des services adaptés aux besoins des enfants continue de peser sur les familles et les agences de services d'aide à l'enfance des Premières Nations. Or, les demandes individuelles de services gouvernementaux qui tiennent compte des besoins particuliers des enfants des Premières Nations ne seraient pas nécessaires si les programmes du gouvernement du Canada avaient initialement été conçus en vue de respecter l'intérêt supérieur de l'enfant et l'égalité réelle des enfants des Premières Nations. Comme l'a fait remarquer le Tribunal, une réforme importante des programmes gouvernementaux existants est nécessaire pour qu'ils puissent répondre aux véritables besoins des enfants des Premières Nations ${ }^{116}$. Autrement dit, pour que les services gouvernementaux fournis aux enfants des Premières Nations soient réellement égaux, selon les objectifs de la LCDP, ceux-ci doivent être conçus spécifiquement pour répondre aux besoins particuliers des enfants.

C'est la raison pour laquelle la Société de soutien a élaboré le « Plan de l'Ourson Spirit $»^{117}$. Le « Plan de l'Ourson Spirit » propose une solution globale et intégrée pour assurer l'élaboration et la prestation de services équitables pour les enfants, les jeunes et les familles de Premières Nations ${ }^{118}$. Ainsi, afin d'éviter que les familles et les agences de protection de l'enfance continuent de porter le fardeau de revendiquer des services adaptés, le « Plan de l'Ourson Spirit » offre une feuille de route au Canada pour identifier de manière proactive les iniquités dans ses services offerts aux enfants des Premières Nations, tels que l'éducation préscolaire, l'enseignement primaire et secondaire, la santé et l'eau. Ce plan encourage le gouvernement du Canada à mettre fin aux inégalités dans la prestation de services publics aux Premières Nations, et d'y remédier de façon exhaustive et holistique ${ }^{119}$. Le Plan de l'Ourson Spirit comprend cinq éléments clés. Premièrement, le Plan demande au Canada de se conformer aux décisions du Tribunal en ce qui a trait au financement équitable des services d'aide à l'enfance et à la mise en œuvre du principe de Jordan ${ }^{120}$. Deuxièmement, il fait appel au Parlement pour demander au directeur parlementaire du

115 Cependant, selon une entente conclue par Santé Canada et la Nation Nishnawbe Aski, le Tribunal ordonne que les demandes de services de santé mentale dans les réserves en Ontario soient traitées et financées par les fonds du principe de Jordan. Voir Société de soutien à l'enfance et à la famille des Premières Nations du Canada c. Procureur général du Canada (représentant le ministre des Affaires autochtones et du Nord canadien), 2017 TCDP 7 aux par. 16-17.

L'entente fournit une manière plus systémique pour combler les lacunes dans les services de santé mentale pour les enfants des Premières Nations en Ontario.

116 Supra note 5 au par. 463.

117 Le plan porte de nom de Spirit Bear, un ourson en peluche membre du Conseil tribal de Carrier Sekani. Spirit Bear représente les quelque 165000 enfants des Premières Nations affectés par les pratiques discriminatoires qui ont fait l'objet de la plainte de la Société de soutien et de l'APN auprès du Tribunal ainsi que les milliers d'enfants à travers le Canada qui ont à cœur la réconciliation. Spirit Bear s'est joint à l'équipe de la Société de soutien en 2008 et a assisté depuis à toutes les audiences devant le Tribunal. Il détient un diplôme honorifique de la Faculté de droit d'Osgoode, est membre de la Société honorifique de common law de la Faculté de droit de l'Université d'Ottawa et a été officiellement admis au barreau par l'Association du Barreau Autochtone du Canada. Son CV est disponible en ligne. Voir Société de soutien à l'enfance et à la famille des Premières Nations du Canada, «Le Plan Spirit Bear », en ligne : Société de soutien à l'enfance et aux familles des Premières Nations du Canada <https://fncaringsociety.com/fr/le-plan-de-spirit-bear> .

118 Société de soutien à l'enfance et à la famille des Premières Nations du Canada, "Le Plan de Spirit Bear », en ligne à : Société de soutien à l'enfance et à la famille des Premières Nations du Canada

119 Ibid.

120 Ibid. au par. 1. 
budget d'évaluer les lacunes dans le financement des services destinés aux enfants et aux jeunes des Premières Nations et de proposer des solutions pour y remédier ${ }^{121}$. Troisièmement, le Plan demande au gouvernement de consulter les Premières Nations et collaborer dans la création de plans exhaustifs et holistiques pour assurer l'équité dans les services publics fédéraux destinés aux enfants et aux jeunes ${ }^{122}$. Quatrièmement, le Plan exhorte tous les ministères qui fournissent des services aux enfants des Premières Nations à subir une évaluation approfondie et indépendante afin « d'identifier les idéologies, les politiques et les pratiques discriminatoires pour les régler », et de rendre ces évaluations publiques ${ }^{123}$. Finalement, il demande que tous les fonctionnaires reçoivent une formation obligatoire visant à identifier et à permettre de « s'attaquer aux idéologies, politiques et pratiques qui compromettent la mise en œuvre des appels à l'action de la Commission de vérité et réconciliation. ${ }^{124}$ »"

Le Plan de 1'Ourson Spirit a reçu un vaste soutien au Canada. Par exemple, l'Assemblée des Premières Nations l'a appuyé à l'unanimité lors de l'Assemblée extraordinaire des Chefs ${ }^{125}$, et un projet de loi a été présenté au Manitoba pour proclamer le 10 mai comme la Journée de l'Ourson Spirit ${ }^{126}$. De plus, de nombreux groupes et organismes, dont UNICEF Canada ${ }^{127}$, Kairos ${ }^{128}$ et les membres de la Faculté de droit de Schulich ${ }^{129}$ ont joint leurs voix à toutes celles qui, au Canada, implorent le gouvernement fédéral d'adopter le plan. En revanche, au moment d'écrire ces lignes, le Canada n'a toujours pas accepté d'entreprendre une telle réforme de sa propre initiative.

Cependant, une nouvelle ordonnance rendue par le Tribunal le 6 septembre 2019 pourrait donner au Canada le coup de pouce nécessaire pour l'inciter à agir ${ }^{130}$. En effet, dans son ordonnance plus récente sur les mesures de redressement, le Tribunal dénonce le Canada qui était conscient des effets néfastes de sa définition étroite du principe de Jordan, mais qui n'a quand même pas agi ${ }^{131}$. Réitérant ses décisions

121 Ibid. au par. 2.

122 Ibid. au par. 3.

123 Ibid. au par. 4.

124 Ibid. au par. 5.

125 Assemblée des Premières Nations, Résolution n 92/2017, « Soutien au Plan de l'Ourson Spirit pour mettre fin aux inégalités dans tous les services publics financés par le gouvernement fédéral qui sont destinés aux enfants, jeunes et familles de Premières Nations », Assemblée extraordinaire des Chefs, Gatineau : le 5, 6 et 7 décembre 2017, en ligne à : Société de soutien à l'enfance et aux familles des Premières Nations du Canada

$<$ https://fncaringsociety.com/sites/default/files/2017\%20AFN\%20Resolutions\%2092\%20SB\%20Plan.pdf $>$ ou en français au https://www.afn.ca/uploads/Social_Development/92-2017_fr.pdf

126 PL 230, Loi sur la Journée de l'ourson Spirit Bear, 4e sess, 41e lég, Manitoba, 2019 (première lecture le 8 avril 2019 ) en ligne : Assemblée législative du Manitoba <https:/web2.gov.mb.ca/bills/41-4/b230f.php\#Note explicative>.

127 Voir UNICEF Canada, « Le budget fédéral prévoit de nouveaux investissements prometteurs dans les enfants au Canada et dans le monde » (19 mars 2019), en ligne UNICEF < https://www.unicef.ca/fr/press-release/le-budget-federal-prevoitde-nouveaux-investissements-prometteurs-dans-les-enfants-au $>$

128 KAIROS, « Kairos urges you to support the Spirit Bear Plan » (3 novembre 2017), en ligne : Kairos Canada $<$ https://www.kairoscanada.org/kairos-urges-support-spirit-bear-plan>.

129 Janet Doucet, « Schulich Law students, staff, and faculty support Jordan's Principle and the Spirit Bear Plan on Bear Witness Day 2018, (10 mai 2018), en ligne : Dalhousie University <https:/www.dal.ca/faculty/law/newsevents/news/2018/05/10/schulich_law_students_staff_and_faculty_support_jordan_s_principle_and_the_spirit_bear_p lan_on_bear_witness_day_2018.html>.

130 Société de soutien à l'enfance et à la famille des Premières Nations du Canada et al. c. Procureur général du Canada (pour le ministre des Affaires indiennes et du Nord canadien), 2019 TCDP 39.

131 Ibid. au par. 231. 
antérieures au sujet du principe de Jordan, il conclut que l'inaction du Canada a causé de la douleur et de la souffrance aux enfants de Premières Nations et à leurs familles ${ }^{132}$. Par conséquent, le TCDP ordonne au Canada de verser 20000 \$, soit la somme maximale permise en vertu du paragraphe 53 (3) de la Loi, à chaque enfant de Premières Nations ayant été pris en charge afin d'accéder à un service dont il avait besoin entre décembre 2007 et novembre 2017, ainsi qu'à ses parents ou à ses grands-parents ${ }^{133}$. De surcroît, le Tribunal conclut que les nombreux retards et problèmes dans la mise en œuvre du principe de Jordan signalent que le Canada ne tenait guère compte des conséquences néfastes de son inaction sur les enfants des Premières Nations et leurs familles ${ }^{134}$. Selon le Tribunal, cette conduite constitue donc un acte discriminatoire délibéré et inconsidéré ${ }^{135}$. Le montant qu'il ordonne au Canada de payer équivaut aux dommages-intérêts punitifs dans le contexte civil ${ }^{136}$. La décision est la huitième ordonnance rendue contre le Canada en matière de redressements visant à inciter l'intimé à mettre fin à son traitement discriminatoire envers les enfants des Premières Nations. Le message du Tribunal au Canada ne peut pas être plus clair : la discrimination vous coûtera fort cher.

Il reste à voir si le risque de se faire ordonner de payer de telles sommes dans de futures plaintes de droits de la personne sera suffisant pour forcer le Canada à prendre des mesures proactives pour éliminer la discrimination dans tous les services offerts aux enfants des Premières Nations. Alors que le Canada conteste l'ordonnance de septembre 2019 à la Cour fédérale, le Tribunal demeure saisi de cette plainte portant sur les services d'aide à l'enfance et le principe de Jordan ${ }^{137}$. Il rendra au cours des prochains mois des ordonnances à moyen et à long terme pour donner suite à sa décision de janvier 2016. Si le Canada refuse de mettre en œuvre le Plan de l'Ourson Spirit, les ordonnances pionnières du Tribunal pourraient au moins servir de barème d'évaluation des autres services publics du gouvernement pour les enfants des Premières Nations.

\section{CONCLUSION}

Le gouvernement du Canada a une longue et déshonorante histoire de discrimination à l'égard des enfants des Premières Nations ${ }^{138}$. Le système des pensionnats autochtones, conçu à l'origine par le gouvernement du Canada et l'Église comme un «système scolaire », visait à scolariser, à assimiler et à évangéliser les enfants autochtones. À partir des années 1960, le système s'apparente davantage aux services d'aide à l'enfance, lorsqu'il commence à cibler les orphelins et les enfants dits "négligés ». Quand les derniers pensionnats autochtones dans le pays ferment leurs portes, le Programme des services à l'enfance et à la famille de Premières Nations du gouvernement du Canada voit le jour ${ }^{139}$. Ce programme

132 Ibid. au par. 222 et 223.

133 Ibid. au par. 225.

134 Ibid. au par. 231.

135 Ibid. au par. 234.

136 Ibid. au par. 254.

137 Le 4 octobre 2019, le Canada a déposé un avis de requête en révision judiciaire à la Cour fédérale du Canada. Canada, « Notice of Application for Judicial Review », Attorney General of Canada v First Nations Child and Family Caring Society and al., Court File No. T-1621-19 (4 octobre 2019) en ligne : Société de soutien à l'enfance et aux familles des Premières Nations du Canada <https://fncaringsociety.com/sites/default/files/federal_court_document_t-1621-19.pdf > 138 Supra note 5 aux par. 406 à 412.

${ }^{139}$ Ibid. au par. 413. 
reconduit et exacerbe de nombreux dommages causés par les pensionnats autochtones plutôt que de tenter d'y remédier ${ }^{140}$. De même, le manque de coordination au sein du gouvernement fédéral, et entre ses ministères, ainsi qu'entre les gouvernements provinciaux et territoriaux font souvent en sorte que les enfants des Premières Nations sont laissés en attente de services dont ils ont désespérément besoin, se voient offrir des services inéquitables ou se font refuser des services qui sont pourtant offerts aux autres enfants du Canada. Dans les deux cas, le gouvernement du Canada n'a pas agi dans l'intérêt des enfants des Premières Nations et cela a mené à leur prise en charge de façon disproportionnée par l'État. En effet, lorsque la plainte de la Société de soutien et de l'APN a été déposée en février 2007, environ trois fois plus d'enfants des Premières Nations faisaient l'objet d'un placement qu'il y en avait lorsque le système des pensionnats battait son plein ${ }^{141}$. Lorsque le TCDP a établi que la conduite du Canada portait atteinte à la LCDP, il lui incombait d'ordonner des mesures réparatrices visant à mettre fin à ces pratiques discriminatoires. C'est ce qu'il a fait en rendant son ordonnance en mai 2017.

Cet article a examiné en détail l'ordonnance du TCDP de mai 2017 portant sur la mise en œuvre du principe de Jordan et le contexte dans lequel elle a été rendue. Confronté à un intimé qui n'a pas voulu ou n'a pas su cesser son comportement discriminatoire, le TCDP a ordonné une panoplie de mesures réparatrices visant la mise en œuvre immédiate et intégrale du principe de Jordan afin de prévenir la discrimination. Il reste à voir si des ordonnances supplémentaires obligeant le gouvernement à se conformer au « Plan de l'Ourson Spirit » seront nécessaires pour mettre fin aux iniquités dans les services offerts par le gouvernement du Canada aux enfants des Premières Nations.

\footnotetext{
${ }^{140} \mathrm{Ibid}$. au par. 422.
}

${ }^{141}$ Ibid. au par. 161. 